\title{
COBERTURA DAS CAMPANHAS DE VACINAÇÃO ANTIRRÁBICA ANIMAL
}

\author{
COVERAGE OF RABIES VACCINATION CAMPAIGNS
}

\author{
Murilo Stefan Gebrim $^{\mathrm{a}^{*}}$, Gabriela Camargo Tobias ${ }^{\mathrm{b*}}$, Cristiane Chagas Teixeira ${ }^{\mathrm{c}^{*}}$
}

murilosgebrim@hotmail.com ${ }^{\mathrm{a}}$, gabicamargo22@gmail.com ${ }^{\mathrm{b} * *}$, cc-teixeira@hotmail.com ${ }^{\mathrm{c}^{*}}$

Universidade Estadual de Goiás*, Instituto de Patologia Tropical e Saúde Pública ${ }^{* *}$

Data de submissão: 03/09/2019

Data de Aprovação:11/09/2019

\section{RESUMO}

Objetivo: analisar a cobertura vacinal nas campanhas antirrábica em Senador Canedo, Goiás, Brasil, no período de 2012 a 2017. Materiais e métodos: estudo descritivo, sobre a cobertura de vacinação antirrábica. A população em estudo compreendeu todos os cães e gatos que foram vacinados no período de 2012 a 2017. As informações foram obtidas pelo DATASUS e os dados foram organizados e analisados em Programa Microsoft ${ }^{\circ}$ Excel. Resultados: no período estudado a cobertura vacinal total do intervalo de 2012 a 2017 foi de 88,5\% para cães e $88,1 \%$ para gatos. O ano de maior cobertura vacinal foi o de 2012 para cães e 2017 para gatos, com 112,2\% e 105\%, respectivamente, onde as metas estabelecidas foram ultrapassadas. Conclusão: diante da letalidade de $100 \%$ dos casos da raiva e o alto custo na assistência preventiva às pessoas expostas ao vírus, torna-se essencial o esforço das secretarias municipais de saúde para realizarem as campanhas vacinais para eliminação e controle deste agravo.

Palavras-chaves: Vacinas antirrábicas; imunização; notificação de doenças; saúde pública

\section{ABSTRACT}

Objective: to analyze vaccine coverage in rabies campaigns in Senador Canedo, Goiás, Brazil, from 2012 to 2017. Materials and methods: a descriptive study on rabies vaccination coverage. The study population comprised all dogs and cats that were vaccinated in the period from 2012 to 2017 . The information was obtained by DATASUS and the data were organized and analyzed in Microsoft ${ }^{\oplus}$ Excel Program. Results: in the period studied the total vaccination coverage of the interval from 2012 to 2017 was $88.5 \%$ for dogs and $88.1 \%$ for cats. The year of greatest vaccination coverage was 2012 for dogs and 2017 for cats, with $112.2 \%$ and 105\%, respectively, where the established targets were exceeded. Conclusion: in view of the lethality of $100 \%$ of cases of rabies and the high cost of preventive care for people exposed to the virus, it is essential that municipal health secretariats carry out the vaccination campaigns to eliminate and control this disease.

Keywords: Rabies vaccines; immunization; disease notification; public health 


\section{INTRODUÇÃO}

A raiva é uma doença transmitida ao homem (antropozoonose), por meio da inoculação do vírus presente na saliva do animal raivoso (infectado com o vírus). A inoculação do vírus no homem ocorre, principalmente, pela mordedura e/ou arranhadura, mas também por lambeduras e quando há lesão de continuidade na pele e mucosa. Essa doença, apesar de ser cosmopolita, descrita a vários séculos e apresentar a vacina como prevenção, continua sendo um problema de Saúde Pública humana e animal ${ }^{1}$.

$\mathrm{O}$ vírus tem predileção pelo sistema nervoso central (neurotrópico), podendo causar uma encefalomielite aguda e progressiva, decorrente da replicação viral nos neurônios, que resulta em letalidade de praticamente $100 \%$ dos casos. Além disso, há um alto custo na assistência às pessoas expostas, com a utilização do esquema vacinal com duas ou quatro doses ou quatro doses mais o soro $^{1-3}$.

Apenas os mamíferos são acometidos pelo vírus da raiva. No Brasil, o morcego é o principal responsável pela manutenção da cadeia silvestre, enquanto cães e gatos, continuam sendo fontes de infecção importantes no perímetro urbano ${ }^{1}$.

Em grande parte dos países em desenvolvimento, cerca de $70 \%$ dos casos notificados de raiva humana ainda tem o cão como transmissor. Enquanto nas Américas, os casos de raiva canina apresentaram redução de $90 \%$ nos últimos 10 anos, graças aos Programas de controle da raiva. No Brasil, os últimos casos de raiva em humanos foram transmitidos por morcegos, que hoje é a fonte de infecção mais importante, como por exemplo, as 8 mortes ocorridas no Pará, Brasil, em maio de 2018. Sendo assim, observase que as campanhas de vacinação contra a raiva, principalmente, de cães, sejam bem desenvolvidas e realizadas ${ }^{1-3}$.

As campanhas de vacinação contra a raiva em cães e gatos é a ação de saúde pública que mais contribui para o controle da doença nessas espécies, tendo como consequência a queda e quase erradicação de casos em seres humanos. De acordo com a Organização Mundial da Saúde (OMS), as coberturas vacinais contra a raiva realizadas durante anos, contribui, positivamente, para o controle da raiva. Além disso, o Ministério da Saúde do Brasil recomenda que pelo menos $80 \%$ dos cães e gatos sejam vacinados para afastar a possibilidade da raiva animal ${ }^{1-3}$.

Com isso, há necessidade de aprimorar, adequar e sempre atualizar as estratégias de campanhas vacinais dos animais susceptíveis a raiva, levando em consideração a situação epidemiológica e outros fatores, como os recursos humanos e financeiros, disponibilidade de insumos, densidade populacional e a estrutura político-administrativa vigente do município ${ }^{2}$. Segundo dados da Superintendência de Vigilância em Saúde (SUVISA) em Goiás, Brasil, a população canina e felina em 2017 foi estimada em, aproximadamente, 1.237.000 de animais. Destes 1.124 .909 cães, a meta de vacinação foi $80 \%$ (899.927 cães) e dos 112.368 gatos, com mesma meta (89.894 gatos) ${ }^{4}$.

Em Goiás, Brasil, a última notificação da doença em humanos, transmitida pela variante 2 (cão), ocorreu em 2001, e o último caso de raiva canina, também transmitida pela variante 2 (cão), foi registrado em $2002^{4}$.

O avanço progressivo observado no controle da raiva, graças às campanhas de vacinação antirrábica animal, apontou grande redução nos números de casos de raiva humana e animal, entretanto, ainda existem áreas vulneráveis à circulação da doença ${ }^{1-4}$. Devido a importância da prevenção contra a raiva humana e animal, o conhecimento acerca da abrangência das campanhas de imunização de cães e gatos é de extrema importância para o planejamento e avaliação das ações de controle da doença ${ }^{1-4}$.

Diante da relevância da campanha de vacinação animal, o objetivo desta investigação foi analisar a cobertura vacinal nas campanhas antirrábica em Senador Canedo, Goiás, Brasil, no período de 2012 a 2017.

\section{MATERIAIS E MÉTODOS}

Trata-se de um estudo descritivo, sobre a cobertura de vacinação antirrábica no município de Senador Canedo, Goiás, Brasil, entre os anos de 2012 e 2017. Este intervalo foi escolhido devido ao fato de apresentarem dados disponíveis para consulta pública. 
Senador Canedo é um município pertencente à região metropolitana de Goiânia, Brasil, faz limite intermunicipal com os municípios de Aparecida de Goiânia, Bela Vista de Goiás, Bonfinópolis, Caldazinha, Goianápolis, Goiânia e Leopoldo de Bulhões. Possui área urbana de 248,291 $\mathrm{Km}^{2}$, e aproximadamente, 102.947 habitantes ${ }^{4}$.

A população em estudo compreendeu todos os cães e gatos que foram vacinados no período de 2012 a 2017.

Os dados foram obtidos por meio do Departamento de Informática do Sistema Único de Saúde (DATASUS) e disponibilizados no endereço eletrônico (http://www.datasus.gov. br) ${ }^{5}$. O DATASUS é um órgão da Secretaria de Gestão Estratégica e Participativa do Ministério da Saúde do Brasil, com a responsabilidade de coletar, processar e disseminar informações sobre saúde (tais como, indicadores de saúde, assistência à saúde, informações epidemiológicas e de morbidade, informações sobre a rede de assistência à saúde, estatísticas vitais, informações demográficas e socioeconômicas) ${ }^{5}$.

Os dados sobre o número de notificações do município de Senador Canedo, Goiás, Brasil foram extraídos através de relatórios quantitativos, que não identificam o indivíduo notificado do Sistema de Informação de Agravos de Notificação (SINAN). Este sistema é alimentado a partir da notificação e investigação de casos de doenças e agravos que constam da lista nacional de doenças de notificação compulsória, como é o caso do Atendimento Antirrábico Humano ${ }^{6-8}$.
Os dados obtidos nos relatórios foram organizados em planilhas do Programa Microsoft ${ }^{\oplus}$ Excel, para posterior análise.

Este estudo foi realizado exclusivamente com dados secundários e agregados, de acesso público e em conformidade com a Resolução do Conselho Nacional de Saúde (CNS) nº 466, de 12 de dezembro de $2012^{9}$, atendendo os fundamentos éticos e científicos exigidos. $\mathrm{O}$ projeto foi dispensado de apreciação por Comitê de Ética em Pesquisa (CEP), conforme recomenda.

\section{RESULTADOS}

No período de 2012 a 2017, a média da cobertura vacinal para cães foi de $88,38 \%$, sendo um total de 61.439 doses realizadas (Tabela 1).

O ano de 2012 apresentou a maior cobertura vacinal em cães, e a menor foi no ano de 2014. A meta de vacinação da população canina aumentou para 15.162 cães, nos anos de 2013, 2016 e 2017, sendo que apenas em 2016 e 2017 as metas vacinais foram atingidas.

É importante ressaltar que no ano de 2015 não houve realização de campanha vacinal, devido ao fato do Ministério da Saúde do Brasil não enviar doses de vacinas aos Estados por problemas na adequação dos laboratórios perante a ANVISA, não havendo tempo hábil para entrega e produção da vacina, não sendo essa distribuída para estados e municípios ${ }^{10}$.

Tabela 1 - Cobertura vacinal de antirrábica canina realizada em cães no município de Senador Canedo, Goiás, Brasil (2012 - 2017).

\begin{tabular}{cccc}
\hline Anos & Meta (un) & Doses (un) & Cobertura (\%) \\
\hline 2012 & 11.800 & 13.244 & $112,24 \%$ \\
2013 & 15.162 & 10.966 & $72,33 \%$ \\
2014 & 12.129 & 8.211 & $67,70 \%$ \\
2016 & 15.162 & 14.755 & $97,32 \%$ \\
2017 & 15.162 & 14.263 & $94,07 \%$ \\
\hline Total & 69.415 & 61.439 & $88,38 \%$ \\
\hline
\end{tabular}

Fonte: DATASUS 5 . 
A Tabela 2 mostra a cobertura vacinal antirrábica realizada em gatos. No período estudado foram realizadas 6.116 doses em gatos, com uma média de cobertura vacinal de $88,12 \%$. No ano de 2017 tivemos a maior cobertura de vacinação com $105,07 \%$ e a menor foi no ano de 2014 com 64,93\%. Nos anos de 2013 e 2014 a meta proposta de cobertura vacinal não foi atingida.
Quando comparada, as duas coberturas vacinais, cães e gatos tiveram aumento nos anos de 2016 e 2017, entretanto, não houve alteração no número estabelecido como meta a ser atingida nesses anos.

Tabela 2 - Cobertura vacinal de antirrábica realizada em gatos no município de Senador Canedo, Goiás, Brasil (2012 2017).

\begin{tabular}{cccc}
\hline Anos & Meta (un) & Doses (un) & Cobertura (\%) \\
\hline 2012 & 1.180 & 1.217 & $103,13 \%$ \\
2013 & 1.516 & 1.095 & $72,22 \%$ \\
2014 & 1.212 & 787 & $64,93 \%$ \\
2016 & 1.516 & 1.424 & $93,73 \%$ \\
2017 & 1.516 & 1.593 & $105,07 \%$ \\
\hline Total & 6.940 & 6.116 & $88,12 \%$ \\
\hline
\end{tabular}

Fonte: DATASUS 5 .

A Tabela 3 apresenta o número de notificações de atendimentos antirrábicos humanos realizados no município de Senador Canedo, Goiás, Brasil, nos anos de 2012 a 2017. No período analisado tivemos 5.172 casos, destes, o ano de 2017 teve a maior frequência de notificações $(19,4 \%)$, seguido de 2012 (17,5\%) e 2016 (17,2\%).

Tabela 3 - Número de notificações de atendimento antirrábico humano realizado no município de Senador Canedo, Goiás, Brasil (2012 - 2017).

\begin{tabular}{ccc}
\hline Ano & Número de Casos & $\%$ \\
\hline 2012 & 903 & $17,45 \%$ \\
2013 & 785 & $15,17 \%$ \\
2014 & 794 & $15,35 \%$ \\
2015 & 797 & $15,40 \%$ \\
2016 & 888 & $17,16 \%$ \\
2017 & 1005 & $19,43 \%$ \\
\hline Total & 5.172 & $100 \%$ \\
\hline
\end{tabular}

Fonte: DATASUS 5 .

\section{DISCUSSÃO}

O presente estudo demostrou em relação a campanha de vacinação antirrábica canina, que houve uma diminuição da cobertura vacinal conforme os anos. Isso, provavelmente, é resultado de dados subestimados da população de cães $^{11-}$ ${ }^{14}$, que hoje é calculado pelo índice de $10 \%$ da população humana, ou seja, para cada 10 pessoas um cão deve ser vacinado. $O$ que demonstra a cobertura vacinal acima de $100 \%$ da meta estabelecida ${ }^{11}$. Nos anos subsequentes a 2012, a cobertura vacinal foi atingida apenas em 2016 e 2017.

Com relação a vacinação em felinos, a meta de cobertura vacinal foi atingida em 2012, 2016 e 2017. Para os anos de 2013, 2016 e 2017, assim como, na população canina, houve um aumento 
na meta de vacinação para 1.516 gatos, o que evidencia o aumento na demanda de vacinação para essa população.

O crescimento da população de felinos domésticos vem ocorrendo principalmente em razão dos hábitos da população humana e as novas formas de habitação que proliferam, como por exemplo, edifícios de apartamentos e condomínios horizontais. Recomenda-se que, o dimensionamento da população de felinos seja formulado da mesma forma que é feita para os cães, para que as atividades de controle da raiva com ênfase adequada às duas espécies de animais. Soma-se a isso, a crescente preocupação com a espécie felina baseando-se no fato de que as regiões urbanas vêm se tornado um abrigo usual de morcegos de diferentes espécies, sendo estes, potenciais alvos de caça de felinos ${ }^{14}$.

Cabe ressaltar que a cobertura vacinal antirrábica animal é influenciada por diversos fatores, dentre os quais podemos citar: divulgação midiática, distribuição espacial e de influência dos postos de vacinação ${ }^{11}$, interesse do Estado ${ }^{12}$, e aumento da população animal ${ }^{13}$.

Em Goiânia, Goiás, Brasil, a cobertura vacinal atingida em 2017 foi de $86 \%$ da meta, sendo 153.900 cães e 18.896 gatos vacinados ${ }^{7}$. No estado de Pernambuco, Brasil, no ano de 2017, a meta foi imunizar cerca de 1,2 milhão da população canina e felina e, conseguiram atingir aproximadamente $90 \%$ de cobertura, com 800.000 cães e 300.000 gatos $^{15}$.

Na Paraíba, Brasil, os resultados das campanhas contra raiva mostraram que $o$ percentual de cães vacinados na campanha de 2006, nos municípios brasileiros de Brejo do Cruz (78,29\%), Brejo dos Santos (61,16\%), Jericó $(61,26 \%)$ e São Bento (76,32\%), não atingiram o percentual mínimo recomendado pelos Programas oficiais, que é de $80 \%$ de cobertura vacinal real ${ }^{16}$.

A divulgação da campanha antirrábica animal pelos meios midiáticos como televisão, redes sociais, rádio e "carros de som", são cruciais para a divulgação. Devido à proximidade dos municípios, muitos habitantes costumam levar os animais aos postos de vacinação nas datas em que há maior divulgação da campanha, principalmente, quando essa é feita pelos meios televisionados.
Historicamente, as práticas de comunicação, educação, informação e saúde, desde o início do século XX, estiveram inter-relacionadas e com o objetivo de legitimar os interesses do Estado ${ }^{13}$. Nesse sentido, instrumentos de divulgação de campanha são de extrema influência na efetividade das mesmas.

Conforme os dados apresentados neste estudo, percebemos que a campanha antirrábica, quando realizada em uma mesma data pelos municípios limítrofes, atinge um maior alcance de animais vacinados. Fato constatado nos anos de 2016 e 2017 onde as datas de início, fim e dia " $D$ " da Campanha Antirrábica Animal foram as mesmas, apresentando um quantitativo maior (acima de 90\%), quando comparado aos anos de 2013 e 2014, em que a meta de $80 \%$ não foi atingida.

Neste estudo, em relação aos números de doses aplicadas entre os anos de 2012 a 2017, concluímos que houve queda nos anos de 2013 e 2014. Segundo a Superintendência de Vigilância em Saúde de Goiás, Brasil, os anos de 2012, 2013 e 2014 as datas de início, fim e dia "D" da Campanha Animal ficaram ao cargo dos municípios, por esse motivo não houve conciliação nas datas entre os municípios limítrofes, pois cada Núcleo de Vigilância escolheu a data mais conveniente, ocorrendo então a não conciliação de mesma data.

$\mathrm{O}$ aumento da cobertura vacinal dos cães e gatos também é resultado do crescimento da população humana e animal, considerando a estimativa proposta pela Fundação Nacional de Saúde de um cão para oito habitantes, ou mesmo da proporção de um cão para sete habitantes, recomendada pela Organização Mundial de Saúde (OMS) para países emergentes ${ }^{17-18}$.

Nesse sentido, observamos que a população de Senador Canedo, Goiás, Brasil, aumentou de 84.443 habitantes em 2010, para 105.459 habitantes em 2017. Segundo o Instituto Brasileiro de Geografia e Estatística (IBGE) quando comparado à população de cães em 2010 com, aproximadamente, 10.555 animais e em 2017 com mais de 15 mil cães, percebe-se que também há um aumento considerável ${ }^{19}$.

Em relação as notificações de atendimento antirrábico humano, houve um aumento no período analisado, com maior frequência de notificações em 2017, o que sugere um crescimento 
da população humana e animal no município. Esse aumento no número de notificações pode ser justificado pela maior frequência e ênfase em capacitações, no Protocolo de atendimento realizado pela equipe de Vigilância Epidemiológica e pelo Departamento de Controle em Zoonoses do município.

Além disso, a disponibilidade crescente de cães nas ruas, propicia por sua vez, a reprodução desordenada destes animais, o que aumenta os fatores de risco à população, uma vez que, a Organização Mundial de Saúde (OMS) reforça que um dos meios de se evitar a transmissão de zoonoses (raiva) consiste no controle da população canina $^{20}$.

O fato de ter animais em ambientes de rua, sobretudo, cadelas e filhotes, proporciona um maior grau de risco para agressões a seres humanos, principalmente, devido ao instinto maternal e protetor do animal. Existem diferentes classificações das motivações de ataques e ferimentos por animais, entre eles, dominância, medo, dor, brincadeiras, idiopática, intersexual, aprendida, maternal, médica, protetora, predatória e redirigida ${ }^{14}$.

Sendo assim, a vacinação animal é de tamanha importância devido os animais domésticos serem os principais causadores de notificações de atendimento antirrábico humano e pelo fato que essa doença apresenta letalidade em aproximadamente $100 \%$ dos casos confirmados, além do alto custo na assistência dos indivíduos expostos $^{21}$, o que gera impacto econômico considerável ${ }^{22}$.

Em Senador Canedo, Goiás, Brasil, não houve casos confirmados de raiva humana ou animal em perímetro urbano no período estudado ${ }^{6}$. O último caso de raiva humana em Goiânia, Goiás, Brasil, foi notificado em fevereiro de 1999. Porém, no ano de 2012, foram registrados um caso de raiva em gato e dois casos em morcegos e, em 2014, foram registrados um caso em cão e seis casos em morcegos. Lembrando que em cães e gatos a prevenção é feita pela vacina, que pode ser aplicada em animais com mais de três meses de vida ${ }^{8}$.

As agressões por mordedura canina e arranhadura e mordedura felina são os principais agravos que levam o paciente a procurar o atendimento antirrábico, sendo que a severidade da agressão vai desde o acometimento físico de membros do corpo até a riscos biológicos ofertados no momento da agressão.

Atualmente, grande parte da população se dirige aos departamentos de saúde para tratamento adequado da situação. Contudo, mesmo com toda a informação relacionada ao tratamento, ainda é crescente a população de animais semidomiciliados e não domiciliados, que continuarão a oferecer riscos à Saúde Pública, visto a desinformação sobre a guarda responsável desses animais $^{23}$. Dessa maneira, o número de agressões e notificações tendem sempre a aumentarem.

No ano de 2016, houve uma diminuição na utilização da vacina antirrábica humana no esquema de profilaxia da raiva devido à recomendação do Ministério da Saúde do Brasil, de uso racional das doses de vacinas disponíveis ${ }^{24}$. Como resposta a essa recomendação, a observação do estado clínico e do histórico de vacinação antirrábica dos animais tornou-se mais importante, não só para prevenção e controle da raiva animal, quanto à prevenção da raiva humana.

\section{CONCLUSÃO}

Evidenciou-se a realização das campanhas de vacinação antirrábica animal para controle da doença, uma vez que, a letalidade foi estimada em $100 \%$ dos casos, além do alto custo na assistência preventiva às pessoas expostas ao vírus.

O aumento da cobertura vacinal animal e uso racional das doses de vacinas recomendado pelo Ministério da Saúde do Brasil, que prioriza a observação animal, em detrimento do esquema rotineiro de aplicação de duas doses de vacina ou de quatro doses e soro, tendem a diminuir os gastos com profilaxia e tratamento vacinal.

É importante ressaltar que, independente, da cobertura atingida nas campanhas de vacinação a tendência atual é de um aumento no número de notificações de atendimento antirrábico devido ao aumento da população animal.

Outro ponto a ser destacado, é que a utilização de uma data comum entre os municípios limítrofes para a realização do dia "D", resulta em aumento no número de animais imunizados, reduzindo as 
chances de disseminação do vírus rábico entre os animais urbanos e o uso indiscriminado de vacina antirrábicas humanas em casos de tratamento, em virtude de agressão por animais não imunizados ou suspeitos. Sendo assim, as campanhas de vacinação animal em área urbana devem ser incentivadas a seguirem uma mesma data em municípios limítrofes.

\section{REFERÊNCIAS}

1. Ministério da Saúde (BR). Secretaria de Vigilância em Saúde. Departamento de Vigilância Epidemiológica. Normas técnicas de profilaxia da raiva humana / Ministério da Saúde, Secretaria de Vigilância em Saúde, Departamento de Vigilância Epidemiológica. Brasília: Ministério da Saúde; 2014.

2. Reichman, MLAB. Controle de populações de animais de estimação. Reichman, MLAB, Figueiredo ACC, Pinto HBF, Nunes VFP. Manual Técnico do Instituto Pasteur: São Paulo; 2000.

3. Hatz CF, Kuenzli E, Funk M. Rabies: relevance, prevention, and management in travel medicine. Infect Dis Clin North Am. 2012;26(3):739-53.

4. História da Cidade. Senador Canedo - GO [Internet] 2018. [cited 2018 Abr 17]. Available from: https://www. senadorcanedo.go.gov.br/a-cidade/historia-da-cidade/

5. DATASUS - Departamento de Informática do Sistema Único de Saúde. Brasília: Ministério da Saúde [Internet] 2018. [cited 2018 Abr 17]. Available from: http://www2. datasus.gov.br/DATASUS/index.php?area=02

6. SINAN - Sistema de Informação de Agravos de Notificação. Brasília: Ministério da Saúde [Internet] 2018. [cited 2018 Abr 17]. Available from: http://www. portalsinan.saude.gov.br/o-sinan

7. Campanha Nacional de Vacinação Antirrábica. Brasília: Ministério da Saúde [Internet] 2017. [cited 2018 Abr 14]. Available from: http://pni. datasus.gov.br/consulta_antirabica_17_selecao. asp? enviar $=\mathrm{ok} \& \mathrm{sel}=\mathrm{doses} 01 \& \mathrm{UF}=\mathrm{GO}$

8. Campanha de Vacinação contra a raiva. Goiânia: Superintendência de Vigilância em Saúde [Internet] 2018. [cited 2018 Abr 14]. Available from: http://www. visa.goias.gov.br/post/ver/223870/campanhade-vacinacao-contra-raiva-sera-nesse-sabadodia-23-09

9. Ministério da Saúde (BR). Conselho Nacional de Saúde. Resolução $\mathrm{n}^{\circ}$ 466, 2012. Diretrizes e Normas regulamentadoras de pesquisa envolvendo seres humanos. Brasília: Ministério da Saúde; 2012.
10. Ministério da Saúde (BR). Ofício Circular GVE XIV $n^{0} 185 / 2015$. Declaração do MS de que não tem prazo definido para receber as doses da vacina antirrábica canina/felina. Brasília: Ministério da Saúde; 2015.

11. Moretti GMA. Estudo da campanha de vacinação contra a raiva em cães e gatos em área do município de São Paulo [Dissertação]. São Paulo: Universidade de São Paulo; 2013.

12. Miranda CFJ, Silva JA, Moreira ÉC. Raiva humana transmitida por cães: áreas de risco em Minas Gerais, Brasil, 1991-1999. Cad Saúde Pública. 2003;19(1):91-9.

13. Cardoso JM. Comunicação, saúde e discurso preventivo: reflexos a partir de uma leitura das campanhas nacionais de Aids veiculadas pela TV (1987-1997) [Dissertação]. Rio de Janeiro: Escola de Comunicação-CFCH, Universidade Federal do Rio de Janeiro; 2002.

14. Paranhos NT, Silva EA, Bernardi F, Mendes MCNC, Junqueira DMAG, Souza IOM, et al. Estudo das agressões por cães, segundo tipo de interação entre cão e vítima, e das circunstâncias motivadoras dos acidentes, município de São Paulo, 2008 a 2009. Arq Bras Med Vet Zootec. 2013;65(4):1033-040.

15. Secretaria Estadual de Saúde. Governo do Estado de Pernambuco. PE Vacina Cães e Gatos contra a Raiva, Recife - PE [Internet] 2018. [cited 2018 Abr 14]. Available from: http://portal.saude.pe.gov.br/ noticias/secretaria-executiva-de-vigilancia-emsaude/pe-vacina-caes-e-gatos-contra-raiva

16. Oliveira AVB, Agra da Silva R, Gomes AAB. Campanha Nacional de Vacinação Contra Raiva Animal nos Municípios da Microrregião de Catolé do Rocha - PB, no período e 2007 a 2006. Rev Verde. 2010;5(1):101-09.

17. Ministério da Saúde (BR). Fundação Nacional de Saúde. Encontro Nacional dos Coordenadores de Zoonoses. Relatório Anual. Brasília: Ministério da Saúde; 2002.

18. WHO - World Health Organization. World Society for Protection of Animals. Guidelines for the dog population management. Genebra; 1992.

19. IBGE - Instituto Brasileiro de Geografia e Estatística. Cidades. [Internet] 2010. [cited 2018 Abr 21]. Available from: http://www.cidades.ibge.gov.br/ $\mathrm{xtras} /$ perfil.php? lang $=\& \operatorname{cod}$ mun $=230015 \&$ searc $\mathrm{h}=$ ceara|acarape|infograficos:-informacoes-completas

20. Moutinho FFB, Nascimento ER, Paixão RL. Raiva no Estado do Rio de Janeiro, Brasil: análise das ações de vigilância e controle no âmbito municipal. Ciênc saúde coletiva. 2015;20(2):577-86.

21. Silva MNG, Mistieri MLA, Júnior WSF, Centeno LVP, Pascon JPE, Lubeck I, et al. Projeto "melhor amigo" na conscientização da guarda responsável de animais de estimação. Rev Ciênc Ext. 2013;9(3):43-52. 
22. Saraiva DS, Thomaz EBAF, Caldas AJM. Raiva humana transmitida por cães no Maranhão: avaliação das diretrizes básicas de eliminação da doença. Cad Saúde Colet. 2014;22(3):281-91.

23. Oliveira VMR, Pereira PLL, Silva JA, Miranda CFJ, Rodrigues KO, Rodrigues TO, et al. Mordedura canina e atendimento antirrábico humano em Minas Gerais. Arq Bras Med Vet Zootec. 2012;64(4):891-98.

24. Ministério da Saúde (BR). Nota Informativa $\mathrm{n}^{\mathrm{o}}$ 26-SEI/2017-CGPNI/DEVIT/SVS/MS. Que informa sobre alterações no esquema de profilaxia da raiva humana pós-exposição e orienta quanto à correta utilização da via intradérmica nas situações em que esta for recomendada. Brasília: Ministério da Saúde; 2017.

Como citar este artigo:

Gebrim MS, Tobias GC, Teixeira CC. Cobertura das campanhas de vacinação antirrábica animal. Rev. Aten. Saúde. 2019; 17(61): 13-20. 